\title{
H. Matthys
}

\section{Chronisch obstruktive Lungenerkrankungen - Indikationen für die $\mathrm{O}_{2}$-Langzeittherapie}

\author{
Chronic Obstructive Lung Diseases - Indications for $\mathrm{O}_{2}$-Longterm Therapy
}

\section{Zusammenfassung}

Die chronisch obstruktiven Lungenkrankheiten umfassen im wesentlichen das Asthma und die chronische Bronchitis mit mehr oder weniger Lungenemphysem (COPD). Die Diagnose und Differentialdiagnose geschieht in praxi mittels Anamnese und Lungenfunktionstestmonitoring. Im Gegensatz zum Asthma ist die COPD meist Folge von Tabakrauchen. Die Pharmakotherapie mit langwirksamen $\beta_{2}$-Agonisten und/oder Anticholinergica (Thiotropiumbromid) in Kombination mit inhalativen Steroiden ist bei der COPD weniger effizient als beim Asthma. Lebensverlängernd wirkt bei COPD mit gleichzeitiger chronischer respiratorischer Insuffizienz nur die $\mathrm{O}_{2}$-LZT. Droht im Finalstadium als Folge des Atempumpversagens die $\mathrm{CO}_{2}$-Narkose muss die Dauer$\mathrm{O}_{2}$-Gabe durch eine nichtinvasive Langzeitbeatmung ergänzt werden.

\section{Abstract}

The term chronic obstructive lung diseases sumarizes asthma and chronic bronchitis with more or less emphysema (COPD). Diagnosis and differential diagnosis of asthma and COPD is performed in clinical praxis mainly by anamnesis and lung function monitoring. In contrast to asthma COPD is mostly due to cigarette smoking and may result in chronic respiratory insufficiency. Pharmacotherapy with longacting $\beta_{2}$-agonists (formoterol, salmeterol) and/or anticholinergics (thiotropiumbromid) in combination with inhalative steroids is less effective in patients with COPD than with asthma. Beside cessation of smoking $\mathrm{O}_{2}$-LT is the only evidence based medicine therapy which proved to prolonge live in these patients. In the final stage of the disease with the danger of $\mathrm{CO}_{2}$-narcosis due to respiratory pump failure $\mathrm{O}_{2}$-LT often needs additional noninvasive home ventilation of these patients.
Unter den chronisch-obstruktiven Lungenerkrankungen subsumiert man im Wesentlichen das Asthma bronchiale sowie die chronisch obstruktive Bronchitis und die verschiedenen Emphysemformen [1].

Andere Krankheitsbilder, die mit einer pulmonalen Obstruktion einhergehen, sind das dyskinetische Ziliensyndrom, die Mukoviszidose, die Bronchiolitis obliterans etc., welche im Allgemeinen nicht unter dem Begriff „Chronisch obstruktive Lungenerkrankungen“ subsumiert werden.
Die Diagnose „Asthma“ wird meist lungenfunktionell objektiviert. Sie ist funktionell charakterisiert als eine intermittierende, wechselnd persistierende Atemwegsobstruktion, ausgelöst als Folge einer allergogenen oder nicht allergogenen Provokation mit variabler bronchialer Hyperreagibilität. Vorwiegend Mastzellen und T-Helfer ${ }_{2}$-Lymphozyten, neutrophile und eosinophile Granulozyten kennzeichnen die zugrunde liegende, genetisch determinierte Entzündung der Atemwege (Tab.1).

Für die chronische Bronchitis gilt immer noch die anamnestische WHO-Definition: Husten mit Auswurf an den meisten Tagen während mindestens je 3 Monaten in 2 aufeinander folgenden 
Tab. 1 Auslöser von Asthma bronchiale

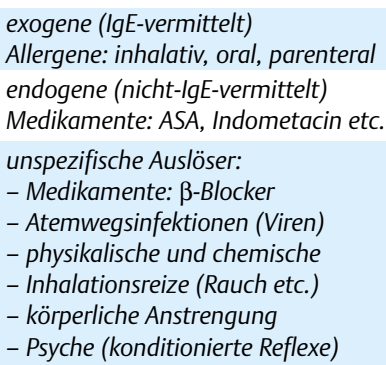

Tab. 2 Chronische Bronchitis

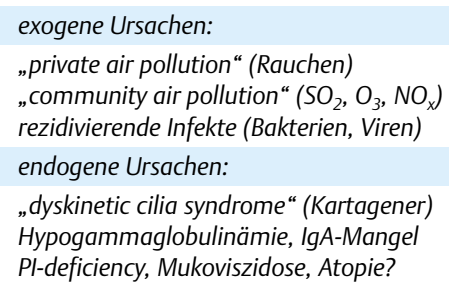

Jahren. Die Ursachen sind vielfältig, wobei die neutrophilen Granulozyten und in der akuten Exazerbation (Steroidindikation) auch die Eosinophilen dominieren (Tab. 2). Elastasenfreisetzung und Antiproteinasenmangel sind nebst genetischen Faktoren für das Fortschreiten der Erkrankung, insbesondere der dadurch bedingten Emphysemgenese, maßgebend.

Das Emphysem ist nach morphologisch-anatomischen Gesichtspunkten als „irreversibler Lungenparenchymverlust, einhergehend mit einem alveolaren Oberflächen- und elastischen Retraktionskraftverlust des Lungengewebes, definiert.

Diese drei Krankheitsbilder werden in der klinischen Routineuntersuchung mittels Ganzkörperplethysmograhie, CO-Diffusionskapazitäts- und arterieller Blutgasmessung schweregradmäßig eingeteilt.

Gelingt es, die ganzkörperplethysmographisch objektivierte pulmonale Obstruktion (Abb.1) durch eine akute ( $\beta_{2}$-Reversibilitätstest) oder länger dauernde pharmakotherapeutische Maßnahme (Kortikoid, $\beta_{2}$ - und/oder Anticholinergika-Reversibiltätstest) teilweise oder ganz reversibel zu gestalten, so liegt eine asthmatische oder bronchitische Atemwegsobstruktion vor. Bei pharmakotherapeutisch irreversibler Atemwegsobstruktion mit Lungenüberblähung und erniedrigtem Einatemzug-CO-Transfer ( $\left.T_{L C O}\right)$ handelt es sich um ein Lungenemphysem, wobei das Helium-single-breath-Volumen in maximaler Inspirationslage stets kleiner als die ganzkörperplethysmographisch gemessene Totalkapazität ausfällt.

Die Differenzialdiagnose zwischen Asthma und Chronischer Bronchitis lässt sich im Carbachol-Provokationstest realisieren, indem bei Asthma die dosisabhängige Bronchokonstriktion im Verlauf der Zeit variabel ausfällt, währenddessen sie bei der
PULMONALE OBSTRUKTION $\left(\right.$ FEV, IVC $\downarrow$, PEF $\downarrow ; \operatorname{TLC} \uparrow$, RV/TLC $\left.\uparrow, \mathbf{R}_{m} \uparrow\right)$

ANTIOBSTRUKTIVE PHARMAKOTHERAPIE

Reversibel, $\mathrm{T}_{\text {Lee }} \mathrm{n}$ Reversibel, $\mathrm{T}_{\text {Lee }} \mathrm{n}$ Irreversibel, $\mathrm{T}_{\text {Lea }}+$

NORMALE LUNGENFUNKTION : PROVOKATIONTESTS

\begin{tabular}{|c|c|c|}
\hline positiv/variabel & negativ/konstant & negativ/konstant \\
\hline 1 & & 10 \\
\hline $\begin{array}{l}\text { ASTHMA } \\
\text { extrinsisch:Atopie, lgEt } \\
\text { intrinsisch:Mscikation? } \\
\text { Eosinophile Entzundg. }\end{array}$ & $\begin{array}{l}\text { BAONCHITS } \\
\text { Oxidantien u. Elastasen- } \\
\text { Orsrioad, Rauchen (py) } \\
\text { Neutrophile Entzindung }\end{array}$ & $\begin{array}{c}\text { EMPHYSEM } \\
\text { Protesseninhỉoinoren- } \\
\text { Mangel, CT: } \\
\text { zertrit, panlobular }\end{array}$ \\
\hline
\end{tabular}

Abb. 1 Die Differenzialdiagnose „Asthma“, „chronische Bronchitis“ und "Lungenemphysem“ geschieht in erster Linie mittels Lungenfunktionstests

chronischen Bronchitis weitgehend negativ oder konstant positiv gemessen wird. Das Letztere gilt auch für das Lungenemphysem, dessen Diagnose sich aber meist schon aus der Kurz- und Langzeit-Irreversibilität der Atemwegsobstruktion unter Pharmakotherapie ergibt. Ein normaler CO-Transferfaktor schließt ein wesentliches Lungenemphysem aus.

Unter COPD (Chronic obstructive pulmonary disease) versteht man die Kombination von den anamnestisch zu erhebenden Symptomen der chronischen Bronchitis mit den funktionellen und evtl. auch radiologischen (CT) Zeichen des Lungenemphysems. Die COPD wird nach den WHO-Prognosen weltweit von der 5. auf die 3. Stelle nach Herzversagen und Schlaganfällen in der Statistik der Todesursachen vorrücken. Hauptursache für das Zunehmen der COPD ist nicht die größer werdende Umweltverschmutzung oder die Staubexpositionszunahme am Arbeitsplatz, sondern die wachsende Zahl nikotinsüchtiger Raucher. Es erstaunt daher nicht, dass die COPD eine Präkanzerose und die chronische Raucher-Bronchitis Hauptursache für den häufigsten, zum Tode führenden Krebs, das Bronchialkarzinom, ist.

Wir können in diesem Zusammenhang festhalten: Die Raucherpathologie ist im Wesentlichen irreversibel. Chirurgische und pharmakotherapeutische Ansätze sind im Vergleich zur Nikotinabstinenz bedeutungslos. Das Inhalationsrauchen ist und bleibt der größte vermeidbare gesundheitliche Risikofaktor, den wir kennen. Die $\mathrm{O}_{2}$-Langzeittherapie ist die einzige lebensverlängernde therapeutische Maßnahme bei fortgeschrittener COPD mit $\mathrm{O}_{2}$-bedürftiger respiratorischer Insuffizienz [2].

\section{Indikationen zur $\mathrm{O}_{2}$-Langzeittherapie (LZT)}

Die $\mathrm{O}_{2}$-LZT wird heute als rein symptomatische Therapie bei allen Formen von Herz-, Lungen und/oder Atempumpversagen induzierter respiratorischer Insuffizienz angewendet, wobei man sich aufgrund der Studien der 80er Jahre, welche nach Evidencebased-medicine-Kriterien bei Patienten mit COPD eine Lebensverlängerung und Lebensqualitätverbesserung nachgewiesen haben, richtet [3-5]. 


\section{Respir. Insuffizienz}
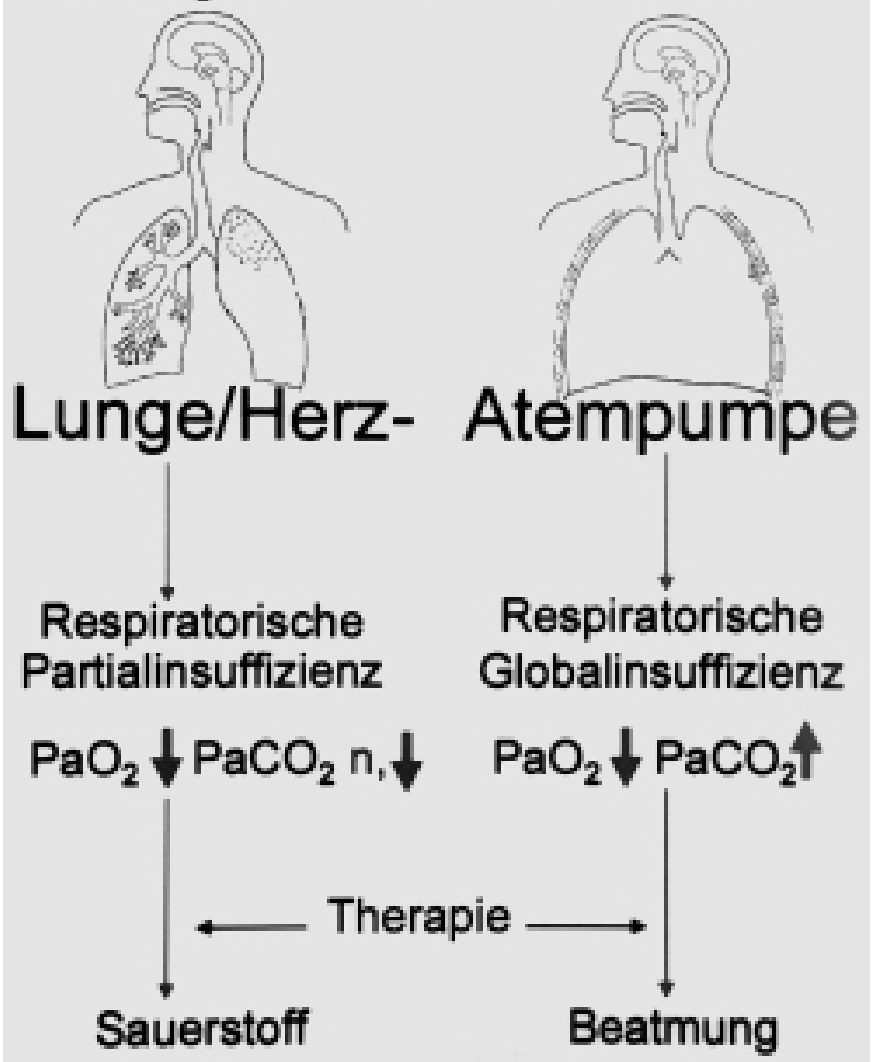

\section{Kombinationen}

Abb. 2 Die Atempumpe umfasst alle Strukturen, welche an der Ventilation beteiligt sind außerhalb der Atemwege und der Lunge. Herzfehler und Herzmuskelversagen („Herzpumpenversagen“) können ebenfalls zu einer respiratorischen Partialinsuffizienz führen. Jedes Versagen der Atempumpe, das anfallende $\mathrm{CO}_{2}$ adäquat abzuatmen, führt zur respiratorischen Globalinsuffizienz.

\section{Wirkungen der $\mathrm{O}_{2}$-Atmung}

Es gilt, sauber zwischen der Indikation für eine akute $\mathrm{O}_{2}$-Gabe und der $\mathrm{O}_{2}$-LZT zu unterscheiden. Eine $\mathrm{O}_{2}$-LZT ist stets indiziert, wenn der $\mathrm{O}_{2}$-Partialdruck drei Monate lang unter $55-60 \mathrm{~mm} \mathrm{Hg}$ liegt. Dabei sollte berücksichtigt werden, dass der $\mathrm{CO}_{2}$-Partialdruck auf $40 \mathrm{~mm} \mathrm{Hg}$ standardisiert wird. $\mathrm{PaO}_{2}$ standardisiert $=\mathrm{PaO}_{2}$ gemessen $-1,66 \times\left(40-\mathrm{PaCO}_{2}\right.$ gemessen $)$. Dieses Vorgehen ist sinnvoll, um Patienten, welche durch Hyperventilation und daher erhöhte Atemarbeit versuchen, ihren $\mathrm{O}_{2}$-Partialdruck über die Grenze der $\mathrm{O}_{2}$-Bedürftigkeit anzuheben. Sie sind meistens dyspnoeischer als Patienten, welche durch relative Hypoventilation, d.h. erhöhten arteriellen $\mathrm{CO}_{2}$-Parialdruck, ihren $\mathrm{O}_{2}$-Partialdruck überproportional senken, um Atemleistung zu sparen. Die $\mathrm{O}_{2}$-Gabe erlaubt den meisten Patienten, ihre Atemarbeit zu reduzieren. Hierbei gilt es abzuklären, ob die $\mathrm{O}_{2}$-Gabe allein nicht zu Somnolenz oder sogar zu einem Narkose verursachenden $\mathrm{PaCO}_{2}$-Anstieg führt. Resultiert die $\mathrm{O}_{2}$-Gabe in einer ungenügenden Ventilation mit kritischem $\mathrm{CO}_{2}$-Anstieg, so darf die $\mathrm{O}_{2}$-Gabe auf keinen Fall entzogen werden, da dadurch der Patient der Gefahr ausgesetzt wird, an $\mathrm{O}_{2}$-Mangel zu versterben oder mindestens einen hypoxischen Hirnschaden zu erleiden. In allen

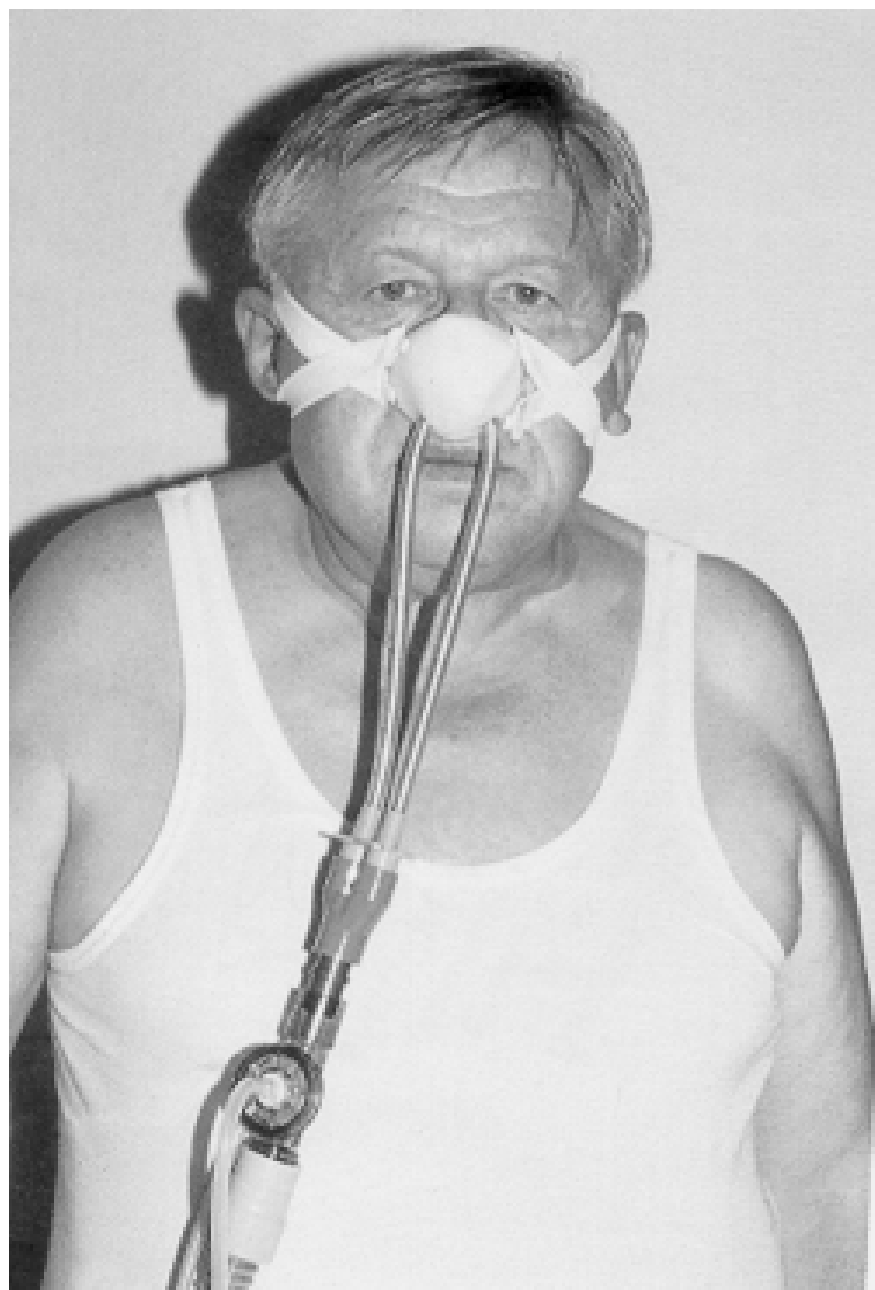

Abb. 3 Patient mit individuell angefertigter Nasenmaske zur intermittierenden Selbstbeatmung in Kombination mit $\mathrm{O}_{2}$-LZT bei chronisch respiratorischer Globalinsuffizienz als Folge von COPD mit Atempumpversagen mit besonderer Dekompensationsgefahr ( $\mathrm{CO}_{2}$-Narkose) nachts respektive im Schlaf.

Fällen mit unter $\mathrm{O}_{2}$-Gabe ungenügender Ventilation muss daher sofort eine nicht invasive oder invasive Beatmung unter fortgesetzter $\mathrm{O}_{2}$-Gabe eingeleitet werden (Abb. 2). Bei Patienten mit so genannter stabiler respiratorischer Globalinsuffizienz, d.h. mit nicht kritisch erhöhtem $\mathrm{CO}_{2}$-Partialdruck, unter kontinuierlicher $\mathrm{O}_{2}$-Gabe konnte in den einschlägigen prospektiv randomisierten Studien gezeigt werden, dass die so genannten „Blue bloater“" gegenüber den normo- und hypokapnischen „Pink-puffer“-Patienten einen Überlebensvorteil genießen, da die $\mathrm{O}_{2}$-Gabe bei relativer Hypoventilation die Atempumpe entlastet und damit der Atemmuskulatur erlaubt, sich zu erholen, ebenso wie die intermittierende Selbstbeatmung (Abb. 3).

Die $\mathrm{O}_{2}$-LZT hat viele positive Wirkungen auf die $\mathrm{O}_{2}$-Transportkette [6 - 11] durch Verminderung von:

- Atemantrieb $\left(\mathrm{PaO}_{2}\right.$-Anstieg $)$

- Atemleistung (Totraumreduktion, Atempumpleistungsreduktion)

- Herzleistungsreduktion $\left(\mathrm{O}_{2}\right.$-Gehaltserhöhung $\rightarrow$ HZV-Erniedrigung)

- Blutviskositätserniedrigung durch Hämoglobingehaltsnormalisierung 
- Lungenstrombahneinengung durch Beseitigung der alveolären Hypoxie mit Rechtsherzentlastung über v. Euler-Liljestrand-Reflex als Akut- und Gefäßremodelling als Langzeiteffekt

- Herzrhythmusstörungen durch Verminderung supra- und ventrikulärer Extrasystolen (Abb. 4)
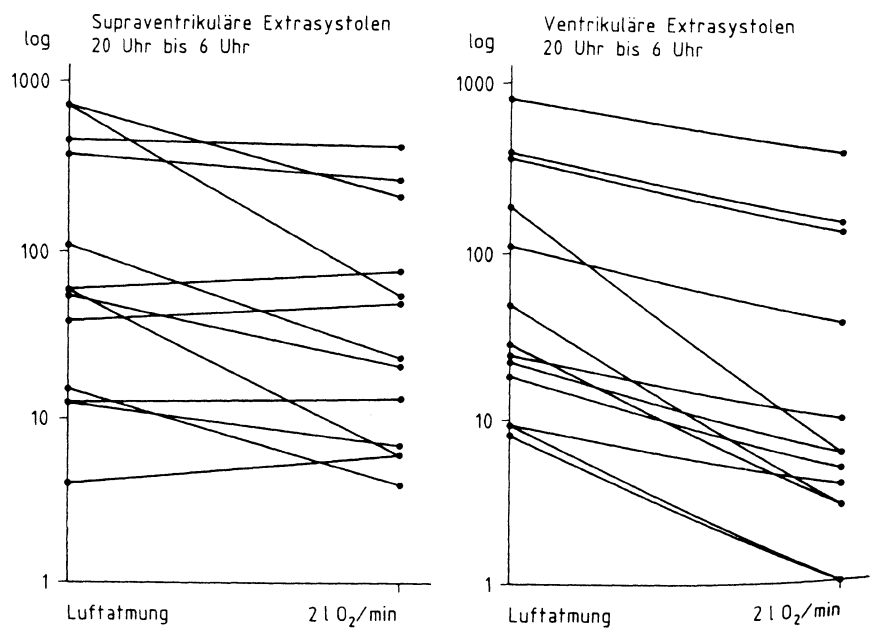

Abb. 4 Abnahme der supraventrikulären und ventrikulären Extrasystolen unter nächtlicher $\mathrm{O}_{2}$-Gabe von $2 \mathrm{I} \mathrm{O}_{2} / \mathrm{min}$ im Gegensatz zur Luftatmung über Nasenbrille.

Folgen der optimierten Gewebsoxigenation durch Anhebung des $\mathrm{O}_{2}$-Partialdruckes in der Einatmungsluft und des $\mathrm{O}_{2}$-Transportvermögens des Blutes $\left(\mathrm{O}_{2}\right.$-Gehalt) sind auch die Verbesserung der Schlafqualität (Dauer - s. Abb. 5 -, REM-Stadium, Stadium II, III und IV) und der körperlichen Leistungsfähigkeit (Gehstrecke) sowie, last but not least, der intellektuellen Fähigkeiten(Vigilanz).

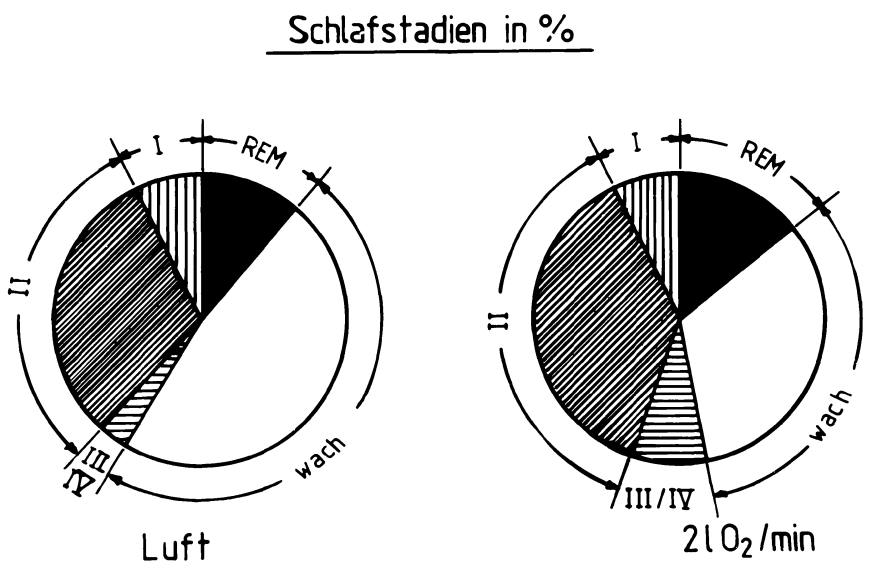

Abb. 5 Schlafstadienverteilung ohne (links) und mit (rechts) 2 I $\mathrm{O}_{2} /$ min. Unter $\mathrm{O}_{2}$ kommt es zu einer Zunahme der Schlafstadien REM, III und IV (Tiefschlaf) und zu einer Abnahme des Wachanteils.

\section{$\mathbf{O}_{2}$-Atmungsdauer}

Sie sollte möglichst über 24 Stunden ununterbrochen erfolgen, da der $\mathrm{O}_{2}$ nur so lange wirkt, als er geatmet wird. $\mathrm{O}_{2}$-Gaben von weniger als 12 Stunden/Tag wirken nicht mehr lebensverlängernd. Die $\mathrm{O}_{2}$-Partialdrücke oder besser: der $\mathrm{O}_{2}$-Gehalt des Blutes sollte nach Möglichkeit bis in den Normbereich angehoben werden.

\section{$\mathrm{O}_{2}$-Dosierung}

Sie wird nach 20-minütiger treppenförmiger Anhebung der inspiratorischen $\mathrm{O}_{2}$-Zufuhr von 1 auf 2 und 31 pro min (so genannte Sauerstofftreppe) blutgasanalytisch aus dem hyperämisierten Ohrläppchen vorgenommen. Pulsoximetrische Messungen können bei wiederholten Kontrollen genügen, sie erlauben aber keine Erfassung des $\mathrm{CO}_{2}$-Partialdruckes. Kontrolluntersuchungen im Schlaflabor oder mittels transkutanen Screening-Geräten können zur Klärung einer nächtlichen Hypoventilation mit oder ohne Apnoephasen und begleitendem Schlafapnoe-Syndrom notwendig werden.

\section{$\mathbf{O}_{2}$-Applikation}

Die $\mathrm{O}_{2}$-Behandlung erfolgt meist über Nasenbrillen mit oder ohne inspirationsgesteuerte $\mathrm{O}_{2}$-Sparsysteme. Letztere sind vor allem bei Flüssig- $\mathrm{O}_{2}$-Gabe indiziert, um $\mathrm{O}_{2}$ zu sparen.

Am wirksamsten ist das Legen eines Minitrachealkatheters oder die Gabe von $\mathrm{O}_{2}$ über eine bereits bestehende Trachealkanüle. Letztere Maßnahmen sind vor allem bei den Patienten in Erwägung zu ziehen, welche über Nasensonden nicht ausreichend oxigeniert werden können. Gesichtsmasken sind im Allgemeinen für die $\mathrm{O}_{2}$-LZT ungeeignet.

\section{$\mathrm{O}_{2}$-Quellen, stationäre und tragbare Geräte}

a) $\mathrm{O}_{2}$-Konzentratoren sind die ökonomischste Art, $\mathrm{O}_{2}$ zu verschreiben. Allerdings kommen sie nur infrage für Patienten, die nicht mehr als $31 \mathrm{O}_{2} / \mathrm{min}$ benötigen und nicht mehr mobil sind, d.h. die sich stets zu Hause aufhalten. Neue Systeme, welche aus Konzentratoren $\mathrm{O}_{2}$ in Flaschen abfüllen lassen für die mobile $\mathrm{O}_{2}$-Heimtherapie haben sich bis jetzt noch nicht durchgesetzt.

b) $\mathrm{O}_{2}$-Flüssigsysteme sind heute bei allen Patienten, die mobil sind sowie tagsüber und nachts und bei körperlicher Belastung $\mathrm{O}_{2}$ benötigen, bevorzugte $\mathrm{O}_{2}$-Quelle. Sie erlauben einen maximalen Aktionsradius und bei entsprechender Beachtung der Logistik in verschiedenen Ländern auch größere Reisen.

c) $\mathrm{O}_{2}$-Gasdruckflaschen werden bei Patienten mit $\mathrm{O}_{2}$-Sparsystemen mit Ein- bis Zweiliterflaschen sowie bei Patienten für kurze Aufenthalte außer Haus eingesetzt. Als stationäre $\mathrm{O}_{2}$-Quelle sind Gasdruckflaschen zu teuer und logistisch zu aufwändig, da sie schneller leer sind als Flüssigkeitstanks. Wie bereits erwähnt, wird sich zeigen, ob sich die neuen leichten $\mathrm{O}_{2}$-Flaschen aus Kunststoff oder aus Aluminium, welche über $\mathrm{O}_{2}$-Konzentratoren zu Hause nachgefüllt werden können, zu einer zukunftsträchtigen Alternative zu den etwas schwereren tragbaren Flüssig- $\mathrm{O}_{2}$-Tanks entwickeln.

\section{$\mathbf{0}_{2}$-Befeuchtung}

Sie sollte nur verordnet werden, wenn von Seiten des Patienten, vor allem bei höheren $\mathrm{O}_{2}$-Flüssen, wegen Austrocknung der Schleimhäute über entsprechende Beschwerden geklagt wird. $0,9 \%$ ige sterile Kochsalzlösung ist hierfür geeignet, destilliertes Wasser für Autobatterien ist nicht zu empfehlen, hingegen genügt auch abgekochtes Leitungswasser in den meisten Fällen, um den zu trockenen inspiratorischen $\mathrm{O}_{2}$ ohne Infektionsgefahr zusätzlich anzufeuchten.

\section{Technische Kontrollen}

Sie sollten am besten alle sechs Monate durch die Lieferanten bzw. bei den $\mathrm{O}_{2}$-Füllungsterminen vorgenommen werden. Dabei 
sollte auch überprüft werden, ob sich der $\mathrm{O}_{2}$-Konsum mit der therapeutischen Empfehlung des Arztes deckt. Nichts ist sinnloser als eine wirksame Therapieverschreibung, welche vom Patienten nicht eingehalten, aber vom Kostenträger bezahlt wird (Non compliance ist die teuerste Krankheit unseres Gesundheitswesens).

\section{Ärztliche Kontrollen}

Wir empfehlen, am Anfang in Abständen von drei Monaten in einem entsprechend ausgewiesenen Zentrum Verlaufskontrollen durchzuführen. Dabei sollten nicht nur die Blutgase und der $\mathrm{Hb}$ Gehalt, sondern auch der HbCO-Gehalt überprüft werden, um festzustellen, ob die empfohlene Raucherabstinenz eingehalten wird. Spirometrische oder ganzkörperplethysmographische Kontrollen sind ebenfalls indiziert, vor allem bei Symptomexazerbationen, wo es gilt, auch die Pharmakotherapie entsprechend anzupassen.

Ob Patienten, welche nach längerer $\mathrm{O}_{2}$-LZT aufgrund der Verbesserung des Krankheitsbildes durch Nikotinabstinenz, konsequente Pharmakotherapie und $\mathrm{O}_{2}$-LZT die eingangs erwähnten Kriterien für die Fortführung der Therapie nicht mehr erfüllen, weiter $\mathrm{O}_{2}$ zuführen sollen, ist nach Evidence-based-medicineKriterien noch nicht untersucht. Hier können auch ergometrische Belastungstests helfen, die $\mathrm{O}_{2}$-LZT-Gabe sinnvoll zu adaptieren [12]. Wir diskutieren mit den Patienten jeweils einen Auslassversuch, wobei aufgrund der Pathophysiologie der Lungenkrankheit bei Exazerbationen die $\mathrm{O}_{2}$-LZT doch meistens wieder indiziert ist. Der Übergang von der alleinigen $\mathrm{O}_{2}$-LZT zur Kombination mit der nicht invasiven Langzeit-Selbstbeatmung ist bei uns leider die häufigere Alternative [2,13].

Abschließend sei darauf hingewiesen, dass von der Deutschen Gesellschaft für Pneumologie kürzlich Leitlinien zur $\mathrm{O}_{2}$-LZT publiziert wurden [14]. Es handelt sich um die erste S3-Leitlinie (u. a. evidenzbasierte formale Konsensusfinding) nach AWMF-Kon-

\section{Literatur}

${ }^{1}$ Joos L, Paré, Sandford AJ. Genetic risk factors of chronic obstructive pulmonary disease. Swiss Med Weekly 2002; 132: 27 - 37

2 Pauwels RA, Buist AS, Calverley PMA et al. Global Strategy for the Diagnosis, Management, and Prevention of Chronic Obstructive Pulmonary Disease. Am J Respir Crit Care Med 2001; 163: 1256 - 1276

${ }^{3}$ Noctural Oxygen Therapy Trial Group. Continuous or nocturnal oxygen therapy in hypoxic chronic lung disease. Am Intern Med 1980; 93: $391-398$

${ }^{4}$ Matthys H. Indikationen für Langzeit-(Heim-)Sauerstofftherapie. Prax Pneumol 1981; 35: 471 - 546

${ }^{5}$ Medical Research Council. Long therm oxygen therapy in chronic hypoexemid or pulmonale complicating chronic bronchitis and emphysema. Lancet 1981; 1: 681-686

${ }^{6}$ Klein G, Rühle KH, Matthys H. Longterm oxygen therapy vs. IPPB-therapy in patients with COLD and respiratory insufficiency. Eur J Resp Dis 1986; 69: 409-415

${ }^{7}$ Rühle KH, Müller B, Klein G et al. Polygraphische Nachtmessungen transkutane Blutgase, Pulmonalisdruck, Schlafstadien unter $\mathrm{O}_{2}$-Therapie bei chronisch obstruktiver Ventilationsstörung. Prax Klin Pneumol 1987; 41: 293-298

${ }^{8}$ Rühle KH, Kempf P, Mössinger B et al. Einfluss von Almitrin, einem Chemorezeptorstimulator, auf die nächtliche Hyperkapnie und den pulmonalarteriellen Druck unter $\mathrm{O}_{2}$-Atmung bei chronisch obstruktiver Lungenerkrankung. Prax klin Pneumol 1988; 42: 411 - 414

${ }^{9}$ Rühle $\mathrm{KH}$, Klein G, Köhler D et al. Effekte einer nächtlichen $\mathrm{O}_{2}$-Therapie auf Gasaustausch, Haemodynamik und Schlafstadien bei Patienten mit chronisch obstruktiver Lungenerkrankung. Atemw-Lungenkrkh 1986; 12: 318-323

${ }^{10}$ Rühle KH, Huber G, Klein G et al. Die Beeinflussung nächtlicher kardialer Rhythmusstörungen durch Sauerstofftherapie bei Patienten mit chronisch obstruktiver Ventilationsstörung. Z Kardiol 1983; 604-607

${ }^{11}$ Rühle KH, Köhler D, Huber G et al. Nächtliche Hypoxie und Hyperkapnie bei Patienten mit chronisch obstruktiver Ventilationsstörung. Therapie mit einem peripheren Chemorezeptorenstimulator. Prax Klin Pneumol 1985; 39: 197-200

12 Rühle KH, Schmitz-Schumann M, Matthys H. Ergometrie mit Messung der Blutgase. Sollwerte, Indikationen, Aussagemöglichkeiten. Med Welt 1989; 40: $882-886$

${ }^{13}$ Matthys H, Seeger W Hrsg. Klinische Pneumologie. Berlin, Heidelberg, New York: Springer, 2002

${ }^{14}$ Deutsche Gesellschaft für Pneumologie. Leitlinien zur Langzeit-Sauerstofftherapie. Pneumologie 2001; 55: $454-464$

15 Sitter H, Goeckenjan G, Magnussen H. Der Weg zur ersten S3-Leitlinie in der Pneumologie. Pneumologie 2001; 55: 452-453 